\title{
Review of: "Path planning and smoothing of mobile robot based on improved artificial fish swarm algorithm"
}

Wenyu CAI

Potential competing interests: The author(s) declared that no potential competing interests exist.

Aiming at the path planning and smoothing of mobile robots, this paper proposed an algorithm that integrates the improved artificial fish swarm algorithm with continuous segmented Bézier curves. As we know, the artificial fish school algorithm and the Dijkstra algorithm are more traditional methods.

Therefore, the innovation of this paper is limited. Besides, there are some minor problems. There are three different spellings of Bézier in the text, Bézier Bezier and Bessel, which is correct?

1. When using the abbreviation for the first time, you need to indicate its full name and check whether the abbreviation is correct.

2. Check the full text according to the English writing standard, including punctuation, capitalization, etc.

3. Modify the font format in the image according to the template.

4. The font size in the formula needs to be unified throughout the whole text, and there are errors in the subscripts and subscripts of some fonts.

5. There are errors in the formula reference in the text.

6. Each part of the formula needs to be explained in detail.

7. Why use a third-order Bézier curve instead of a second-order?

8. In page 2, what is FFS?

9. How to get values in (17.4)?

10. Many many spelling mistakes.. 